\title{
O Potencial dos Exergames para desenvolvimento de Atitude Positiva na Educação Física Escolar
}

\author{
Marlom Zotti Bittencourt - ESEFID/UFRGS - marlomctm@gmail.com \\ Eliseo Reategui - PPGIE/UFRGS - eliseoreategui@gmail.com \\ Ricardo Radaelli - Instituto Federal Farroupilha - ricardoradaelli@gmail.com \\ João B. Mossmann - PPGIE/UFRGS - mossmann@gmail.com
}

\begin{abstract}
Resumo: Com o advento dos Exergames, novas possibilidades de combinação entre jogos digitais e exercício físico foram estabelecidas. Este novo paradigma desencadeou o desenvolvimento de diversos estudos favoráveis ao uso destes artefatos, incluindo na disciplina de Educação Física escolar. Neste trabalho, buscou-se compreender de que maneira um laboratório com estes jogos poderia contribuir ao desenvolvimento de uma atitude positiva frente à Educação Física. No estudo realizado, a atitude de um grupo de 28 alunos foi avaliada considerando aspectos cognitivo e afetivo, em uma intervenção de 20 semanas. Após este periodo, todos alunos responderam a Pesquisa sobre Atitude com Relação à Educação Física (SATPES). Os resultados mostraram que os exergames tiveram boa aceitação, provendo novas possibilidades de desenvolvimento de atividades fisicas envolvendo a tecnologia.
\end{abstract}

Palavras-chave: Exergames, Jogos Digitais, Componente Atitude

Abstract: The introduction of exergames brought new possibilities to combine games and physical exercise. This new paradigm triggered the development of several studies favorable to the use of exergames in Physical Education. In this work, we tried to understand how an exergame lab with these games could contribute with the development of positive attitude towards school Physical Education. In the study carried out, the attitude of a group of 28 students was evaluated, considering cognitive and affective aspects, in activities during 20 weeks. After this period, all students answered the Student Attitude Toward Physical Education Survey (SATPES). Results showed that exergames had a good acceptance among students, providing new possibilities for practicing physical activities using technology.

Keywords: Exergames, Digital games, Attitude component

\section{Introdução}

Os Exergames, ou videogames ativos, inauguraram um novo mundo de possibilidades de interação corporal que combina a dimensão dos jogos digitais com exercício físico. Este novo paradigma resultou em um ponto de inflexão na associação dos videogames à atividade física, desencadeando o desenvolvimento de diversos estudos favoráveis ao uso dos exergames. Tais estudos apontam os benefícios deste tipo de jogo em diversos aspectos relacionados à saúde, como aumento no nível de atividade física (Mhurchu et al., 2008) efeitos na composição corporal (Maddison et al., 2011), aumento no dispêndio energético (Graves et al., 2007). A partir destes resultados, passou a competir à Educação Física escolar investigar de que maneira esses jogos podem ser incorporados em práticas escolares regulares. Neste aspecto, a maior parte dos estudos relacionando exergames e educação física têm focado no potencial destes jogos em melhorar aspectos de saúde dos estudantes, em como envolvê-los em atividades sociais e como melhorar seu desempenho acadêmico (Staiano e Calvert, 
2011). Neste projeto, o enfoque foi diferente ao buscar-se compreender de que maneira os exergames poderiam afetar a atitude dos alunos em práticas regulares da Educação Física. O termo atitude é normalmente utilizado para descrever a disposição de um indivíduo a responder positiva ou negativamente a algo. Atitudes podem desempenhar um papel significativo no aproveitamento do aluno na escola (Ajzen, 2005). Alunos com atitudes positivas em relação têm mais chance de prestar atenção na aula, se esforçar com mais afinco e alcançar notas mais altas, ao passo que alunos com atitudes negativas são mais propensos a não participar das tarefas, exercer um esforço limitado e não atingir os objetivos propostos (Bryan, 2012).

A atitude dos alunos em relação à Educação Física tem sido pesquisada por muito tempo, sendo o currículo um dos principais fatores de impacto neste aspecto (Rikard, 2006). Todavia são poucos os estudos consolidados que relacionam a atitude dos alunos aos exergames em práticas de Educação Física. No contexto da pesquisa aqui proposta, também julga-se importante conhecer as percepções dos alunos para que possam ser avaliadas e consideradas na construção do currículo de Educação Física. Com base nessas considerações, definiu-se a seguinte questão norteadora para a pesquisa: De que maneira práticas regulares em um Laboratório de Exergames pode influenciar a atitude dos alunos em relação à Educação Física?

\section{Os Exergames e a Educação Física}

Com o advento dos exergames, tornou-se possível incorporar os jogos digitais na disciplina de Educação Física, empregado-os como instrumento de apoio pedagógico em alternativa às práticas já estabelecidas da área (Papastergiou, 2009). Mozelius e colaboradores (2014) argumentam que atualmente os alunos gastam mais horas jogando videogames do que lendo livros. Desta forma, por meio dos exergames, é possível fazer com que os alunos pratiquem atividades físicas várias vezes em um ambiente que não lhes é maçante. Ho e colaboradores (2017) desenvolveram estudo que mostrou a aceitação dos participantes quanto ao uso de exergames, destacando que estes são uma alternativa promissora para motivar a prática de exercícios físicos. Esta aceitação é reforçada também por Barg-Walkow et al. (2017) que afirmam que os benefícios destes jogos podem ser desfrutados por pessoas em qualquer idade, introduzindo práticas de atividade física mesmo para indivíduos sedentários.

$\mathrm{Na}$ pesquisa realizada por Trout e Christie (2007), as autoras comentam os potenciais benefícios dos exergames na escola e apresentam cinco exergames populares, assim como propostas concretas de sua exploração pela turma inteira. Nesta mesma direção, Sheehan e Katz (2012) demonstraram a viabilidade da utilização de exergames como recurso prático nas aulas de Educação Física. Seu estudo conduzido com 64 estudantes do $4^{\circ}$ ano durante um período de 6 semanas foi focado no desenvolvimento e avaliação da estabilidade postural. Os autores mostraram que estudantes que trabalharam com os exergames aprimoraram sua estabilidade postural em comparação aos alunos das aulas regulares de Educação Física. Já a pesquisa de Manley e Whitaker (2011) mostrou que o uso de exergames pode ser considerado uma novidade e abordagens de ensino inovadoras podem aumentar o entusiasmo dos estudantes pelo conteúdo da disciplina.

No Brasil, a pesquisa sobre a utilização dos exergames em práticas relacionadas à Educação Física e saúde também tem avançado significativamente. Vaghetti e colaboradores (2011) apresentam um estudo sobre o emprego dos exergames no ciberespaço, e argumentam que os conteúdos da Educação Física podem ser transportados para o meio virtual através dos exergames, possibilitando que conteúdos teórico-práticos da disciplina sejam trabalhados a distância. A pesquisa realizada 
destacou também que o emprego dos exergames em práticas de Educação Física requer a formação continuada de professores. Finco e Fraga (2012) realizaram um estudo baseado na etnografia virtual para avaliar de que maneira os exergames poderiam incentivar a prática de atividades físicas de maneira lúdica. Três comunidades virtuais foram pesquisadas na busca por relatos dos participantes quanto à influência do jogo em práticas de atividade física e manutenção de uma alimentação saudável. $O$ trabalho realizado possibilitou concluir que os exergames podem contribuir com a prática de atividade física regular por parte dos jogadores, trazendo implicações significativas para a área da Educação Física. Já Baracho e colaboradores (2012), em um estudo com 117 jovens entre 13 e 14 anos, demonstrou o surgimento da tríade televisão-computadorvideogame como importante fonte de comunicação e informação, fator determinante em sua formação e visão de mundo. Neste cenário, para a Educação Física, os exergames assumem um significado distinto ao possibilitar o controle dos jogos pela movimentação corporal, quebrando o paradigma da inatividade dos jogos eletrônicos.

A pesquisa apresentada no presente artigo difere destes outros estudos ao abordar o construto atitude e buscar demonstrar de que maneira os exergames podem contribuir com o desenvolvimento de uma atitude positiva dos alunos em práticas regulares na Educação Física. Por outro lado, os trabalhos aqui descritos convergem no sentido de que podem contribuir para a problematização do uso dos exergames como ferramenta de ensino. Um ponto importante ao introduzir novos recursos na Educação Física escolar é que seja feita uma reflexão aprofundada sobre seu valor educacional, focando não somente no seu valor relacionado ao dispêndio energético. As evidências empíricas para apoiar a efetividade dos exergames na Educação Física são ainda recentes, mas os achados apresentados até o momento apontam para um quadro positivo.

\section{Atitude na Educação Física}

Uma das questões centrais da pesquisa na área da Educação é identificar e compreender os fatores que influenciam o engajamento dos estudantes em atividades propostas. Um destes fatores é a atitude (Bryan, 2012). O termo é normalmente utilizado para descrever a disposição de um indivíduo a responder positiva ou negativamente a um objeto, local, pessoa ou atividade. Trata-se de um construto que, sendo inacessível à observação direta, deve ser inferido a partir de respostas mensuráveis (Ajzen, 2005).

A atitude dos alunos em relação à Educação Física tem sido pesquisada por muito tempo. Subramaniam e Silverman (2007) aplicaram esta noção à área curricular da Educação Física, sugerindo que o fator atitude desempenha um papel vital na disciplina, considerando que atitudes podem influenciar no engajamento e posterior continuidade do aluno em práticas envolvendo atividade física. Estudantes que têm atitudes positivas quanto à Educação Física estão mais aptos a exercer maior esforço, atender às instruções e alcançar sucesso nas tarefas, se comparados a estudantes com atitudes negativas. Em consonância com esta ideia, Chatzisarantis et al. (2005) afirmam que estudantes que relatam atitudes positivas em relação às experiências com atividade física estão mais inclinados a continuar estas atividades na idade adulta do que os que têm atitudes negativas neste âmbito.

No intento de inferir informações sobre atitude, pesquisadores têm abordado o tema por perspectivas diferentes. A maior parte dos estudiosos da Educação Física tende a visualizar a atitude sob o prisma de um único componente, concepção esta que sugere que a atitude é uma decisão pessoal, baseada na "positividade" ou "negatividade" percebida pelo sujeito em relação ao objeto da atitude em questão. Essa visão restringe 
o uso do termo apenas ao componente afetivo, o que consequentemente pode resultar na avaliação de apenas um aspecto do construto atitude. Outros pesquisadores propõem uma visão duplo componente, afirmando que a atitude envolve os aspectos afetivo e cognitivo. Por esta perspectiva, o componente afetivo mensura o grau de atração emocional ou sentimento em relação ao objeto da atitude. Por exemplo, gostar da Educação Física ou da prática de atividade física. Já o componente cognitivo dá conta das crenças acerca das características do objeto da atitude, por exemplo, crer que a participação na Educação Física proporciona melhorias na aptidão física do praticante (Ajzen, 2005). Os domínios cognitivo e afetivo são componentes-chave de como as atitudes são formadas e podem influenciar o desempenho do aluno.

Entre os estudos sobre a atitude dos alunos com relação à Educação Física, em pesquisa realizada com 400 estudantes entre 12 e 14 anos Gomwe (2012) identificou que os aspectos que mais apontavam para atitudes negativas com relação à Educação Física estavam relacionados ao currículo. As respostas dos alunos a um conjunto de questões demonstrou que eles consideravam a Educação Física muitas vezes entediante devido à falta de variedade de atividades propostas pelos professores. Em resultado similar, Luke e Sinclair (1991) solicitaram a 488 alunos que comentassem suas experiências na Educação Física da Educação Infantil até o $2^{\circ}$ do Ensino Médio. A determinante mais influente de atitude, tanto positiva (52,7\%), quanto negativa (39\%), foi o conteúdo do currículo.

Por fim, Ryan, Fleming e Maina (2003) buscaram determinar a atitude de 611 alunos dos anos finais do Ensino Fundamental em relação aos professores e aulas de Educação Física. Os resultados indicaram que os alunos apreciavam ter variedade de atividades $(83 \%)$, gostavam dos professores $(80 \%)$ e se divertiam $(79 \%)$ em suas aulas de Educação Física.

Com base nos achados explicitados nos estudos acima, observa-se que inovação e diversidade na proposta de atividades figuram como fatores importantes que impactam na atitude dos alunos quanto à Educação Física escolar. Contudo, é difícil encontrar evidências de que as percepções dos alunos sejam sistematicamente utilizadas na construção do currículo de Educação Física. Ao invés disso, o delineamento do currículo, incluindo a seleção e duração de implementação de cada conteúdo são comumente orientados pela teoria, e não por dados empíricos.

Embora os estudantes apresentem diferentes atitudes em relação à Educação Física em decorrência de vários fatores como experiências anteriores na disciplina, nível de habilidade, gênero, idade, série escolar, classe social, etnia e deficiências (Subramaniam e Silverman, 2007), estas atitudes podem ser modificadas com base em contextos situacionais, tais como o ambiente da aula, a variedade das atividades e os conteúdos abarcados pelo currículo (Ajzen, 2005).

\section{Metodologia}

Para responder à questão de pesquisa proposta, foi realizado um estudo em uma escola particular em Porto Alegre, Rio Grande do Sul. Foi criado na escola um Laboratório de Exergames, o qual foi estruturado para operar de forma integrada e consoante ao Currículo do Movimento da escola, no intento de contribuir com as aulas por meio de atividades físicas sistematizadas e assistidas com jogos digitais. As aulas regulares de Educação Física aconteciam duas vezes por semana, então os estudantes frequentavam uma vez por semana a aula tradicional e no outro dia participavam do laboratório. As aulas no laboratório tinham duração de 50 minutos e foram conduzidas por 20 semanas. 
A estrutura das aulas foi organizada para oferecer atividades para quatro estudantes por vez. Os alunos jogavam em pares, enquanto dois estudantes jogavam, o outro par observava no intento de dar sugestões ou até mesmo interagir e auxiliar os colegas. A cada rodada ou espaço de tempo determinado, as duplas intercambiavam suas posições. As atividades eram compostas por três jogos comerciais do console Kinect Xbox 360, sendo eles:

- Your Shape Fitness Evolved 2012: título contendo atividades como dança, aeróbica, yoga, corrida, ginástica localizada;

- Kinect Adventures: título tematizado por um ambiente e atividades lúdicas envolvendo movimentos corporais variados, como chutar, pular, esquivar e agachar.

- Kinect Sports: título contendo jogos com uma diversidade de esportes, como futebol, boxe, tênis, vôlei de praia, atletismo e boliche.

Os três títulos foram avaliados como apropriados para todas as idades conforme a Entertainment Software Rating Board (2018), dessa forma adequados para a utilização no ambiente escolar. Durante as aulas no laboratório, os estudantes eram solicitados pelo instrutor a utilizar as várias modalidades de cada jogo, a fim de colocá-los em contato com diferentes jogos, movimentos corporais e intensidade de atividade física. Além da organização da aula e proposição dos jogos, o instrutor sanava dúvidas, mediava possíveis conflitos, explicava e demonstrava as atividades e gestos corporais.

\subsection{Amostra}

A amostra foi composta por 28 alunos $($ meninos $=19$, meninas $=9)$ do $7^{\circ}$ ano do Ensino Fundamental ao $3^{\circ}$ ano do Ensino Médio, divididos em cinco diferentes grupos conforme sua série, de idades de 11 a $17 \operatorname{anos}(\mathrm{M}=13,5, \mathrm{DP}= \pm 1,82)$. A participação dos alunos foi definida por critério de adesão. Os estudantes obtiveram autorização de seus pais/responsáveis para participação na pesquisa, e eles próprios também assinaram um termo de consentimento livre e esclarecido.

\subsection{Coleta de dados}

O instrumento utilizado neste trabalho para avaliar a atitude dos estudantes foi o Questionário de Atitude dos Estudantes em Relação à Educação Física (SATPES), desenvolvido por Subramaniam e Silverman (2000). O instrumento utiliza uma escala Likert de 5 pontos estruturada com os seguintes valores: 1 (concordo totalmente), 2 (concordo), 3 (neutro), 4 (discordo) e 5 (discordo totalmente). Duas variáveis relacionadas ao construto atitude são avaliadas pelo instrumento, correspondendo à satisfação (aspecto afetivo) e utilidade percebida (aspecto cognitivo). O quadro 1 lista as afirmações que compõem o questionário, mostrando o aspecto avaliado ( $\mathrm{S}=$ satisfação, $\mathrm{U}=$ utilidade percebida), o componente relacionado à questão $(\mathrm{C}=$ currículo, $\mathrm{P}=$ professor), e por fim se a questão foi codificada reversamente na análise $(\mathrm{N})$.

Quadro 1: Instrumento SATPES.

\begin{tabular}{|c|c|c|}
\hline & Afirmação & \\
\hline 1 & $\begin{array}{l}\text { As atividades que eu aprendo na Educação Física fazem minha aula de Educação Física } \\
\text { interessante para mim. }\end{array}$ & $\mathrm{S}, \mathrm{C}$ \\
\hline 2 & $\begin{array}{l}\text { As atividades que eu aprendo em minha aula de Educação Física fazem com que } \\
\text { aprender seja desagradável. }\end{array}$ & $\mathrm{S}, \mathrm{C}, \mathrm{N}$ \\
\hline 3 & $\begin{array}{l}\text { As atividades que aprendo em minha aula de Educação Física me deixam empolgado em } \\
\text { relação à Educação Física. }\end{array}$ & $\mathrm{S}, \mathrm{C}$ \\
\hline 4 & Meu professor de Educação Física faz minha aula de Educação Física parecer sem & $\mathrm{U}, \mathrm{P}, \mathrm{N}$ \\
\hline
\end{tabular}




\begin{tabular}{|c|c|c|}
\hline & importância para mim. & \\
\hline 5 & $\begin{array}{l}\text { Eu sinto que as atividades que aprendo na Educação Física fazem minha aula de Educação } \\
\text { Física no laboratório de Exergames desagradável para mim. }\end{array}$ & $\mathrm{S}, \mathrm{C}, \mathrm{N}$ \\
\hline 6 & $\begin{array}{l}\text { Eu sinto que as atividades que aprendo em minha aula de Educação Física são inúteis para } \\
\text { mim. }\end{array}$ & $\mathrm{U}, \mathrm{C}, \mathrm{N}$ \\
\hline 7 & $\begin{array}{l}\text { As atividades que aprendo em minha aula de Educação Física parecem importantes para } \\
\text { mim. }\end{array}$ & $\mathrm{U}, \mathrm{C}$ \\
\hline 8 & $\begin{array}{l}\text { Meu professor de Educação Física faz minha aula de Educação Física parecer importante } \\
\text { para mim. }\end{array}$ & $\mathrm{U}, \mathrm{P}$ \\
\hline 9 & $\begin{array}{l}\text { Meu professor de Educação Física faz minha aula de Educação Física interessante para } \\
\text { mim. }\end{array}$ & $\mathrm{S}, \mathrm{P}$ \\
\hline 10 & As atividades que aprendo em minha aula de Educação Física são úteis para mim. & $\mathrm{U}, \mathrm{C}$ \\
\hline 11 & $\begin{array}{l}\text { Eu sinto que meu professor de Educação Física faz com que aprender na aula de Educação } \\
\text { Física seja divertido para mim. }\end{array}$ & $\mathrm{S}, \mathrm{P}$ \\
\hline 12 & $\begin{array}{l}\text { Eu sinto que meu professor de Educação Física torna a aula de Educação Física desagradável } \\
\text { para mim. }\end{array}$ & $\mathrm{S}, \mathrm{P}, \mathrm{N}$ \\
\hline 13 & Eu sinto que as atividades que aprendo na aula de Educação Física são valiosas para mim. & $\mathrm{U}, \mathrm{C}$ \\
\hline 14 & $\begin{array}{l}\text { As atividades que aprendo em minha aula de Educação Física parecem sem importância para } \\
\text { mim. }\end{array}$ & $\mathrm{U}, \mathrm{C}, \mathrm{N}$ \\
\hline 15 & $\begin{array}{l}\text { Meu professor de Educação Física faz com que aprender na aula de Educação Física seja } \\
\text { desagradável para mim. }\end{array}$ & $\mathrm{S}, \mathrm{P}, \mathrm{N}$ \\
\hline 16 & $\begin{array}{l}\text { Eu sinto que meu professor de Educação Física faz com que aprender em minha aula de } \\
\text { Educação Física seja valioso para mim. }\end{array}$ & $\mathrm{U}, \mathrm{P}$ \\
\hline 17 & $\begin{array}{l}\text { Eu sinto que meu professor de Educação Física torna minha aula de Educação Física valiosa } \\
\text { para mim. }\end{array}$ & $\mathrm{U}, \mathrm{P}$ \\
\hline 18 & $\begin{array}{l}\text { Eu sinto que meu professor de Educação Física faz com que aprender em minha aula de } \\
\text { Educação Física seja inútil para mim. }\end{array}$ & $\mathrm{U}, \mathrm{P}, \mathrm{N}$ \\
\hline 19 & Meu professor de Educação Física me deixa empolgado em relação à Educação Física. & $\mathrm{S}, \mathrm{P}$ \\
\hline 20 & $\begin{array}{l}\text { Eu sinto que as atividades que aprendo em minha aula de Educação Física fazem com que } \\
\text { aprender seja divertido pra mim. }\end{array}$ & $\mathrm{S}, \mathrm{C}$ \\
\hline
\end{tabular}

Após a aplicação dos questionários, entrevistas foram realizadas com sete alunos (4 meninos, 3 meninas) selecionados conforme seu resultado no SATPES: três alunos com atitudes localizadas mais ao extremo positivo, dois com atitudes localizadas mais ao extremo negativo e dois com atitudes neutras. A entrevista foi composta por questões abertas semi-estruturadas, gravada em meio digital e posteriormente transcrita. $\mathrm{O}$ objetivo desta etapa da pesquisa foi de esclarecer, explicar e expandir os dados quantitativos obtidos durante a primeira fase de coleta.

\section{Resultados}

Os dados dos questionários foram compilados provendo resultados sobre os diferentes componentes relacionados à atitude dos alunos quanto à Educação Física. A tabela 1 apresenta um apanhado geral da pontuação média obtida a partir dos questionários.

Tabela 1: Média e desvio padrão dos componentes da atitude em uma escala de 0 a 5

\begin{tabular}{lc}
\hline \multicolumn{1}{c}{ Componente } & Média \\
\hline (a) Afetivo (Satisfação) & $4,65 \pm 0,39$ \\
(a.1) Satisfação - Currículo & $4,69 \pm 0,39$ \\
(a.2) Satisfação - Professor & $4,61 \pm 0,49$ \\
(b) Cognitivo (Utilidade percebida) & $4,17 \pm 0,81$ \\
(b.1) Utilidade percebida - Currículo & $4,01 \pm 0,84$ \\
(b.2) Utilidade percebida - Professor & $4,33 \pm 0,79$ \\
(c) Atitude geral & $4,41 \pm 0,57$ \\
\hline
\end{tabular}




\subsection{Satisfação - componente afetivo}

Considerando as questões 1, 2, 3, 5 e 20, todas relacionadas ao aspecto satisfação quanto ao currículo (componente afetivo), verificou-se uma atitude particularmente positiva dos estudantes (média $=4,69$; desvio padrão $= \pm 0,39$ ). Alguns relatos confirmaram este achado, como do aluno $\mathrm{P}$ : "Eu achei as atividades bem interessantes porque uniam o videogame com um modo diferente de prática do esporte em si, formando uma coisa lúdica". O comentário do aluno B também reforça esta posição: "Eu gostei bastante. Não é aquela coisa chata. É que eu não gosto de jogar futebol, fazer os esportes ali fora, mas eu curto bastante jogar videogame. Ai achei uma ideia bem bolada, misturar o exercício físico com o videogame, porque aí une o útil ao agradável". Pesquisas anteriores já demonstravam que a apreciação pela Educação Física está muito ligada ao fator diversão, como no trabalho de Rikard e Banville (2006). No caso dos relatos aqui apresentados, percebe-se que nem todos os estudantes gostam das práticas propostas na disciplina. Desta forma, torna-se importante propor práticas alternativas.

Considerando as questões 9,11,12,15 e 19 referentes ao componente afetivo (satisfação) em relação ao professor, verificou-se também uma atitude positiva dos estudantes (média $=4,61$, desvio padrão $= \pm 0,49$ ), constatação explicitada nas palavras do aluno P: "Eu achei a interação com o professor bem positiva, me senti bem à vontade executando as atividades". Os comentários do aluno M também reforçaram esta posição: "Eu acho que foi bem legal a participação do professor. Ele propôs atividades, tentou fazer um cronograma e de uma forma que conseguia conduzir bem as atividades". A pesquisa de Figley (1985) alinhava-se a estes posicionamentos ao demonstrar que o fator mais frequentemente mencionado em relação às atitudes dos estudantes quanto à Educação Física $(37 \%)$ era o professor, principalmente seu comportamento. Ao unificar os resultados das questões referentes ao componente afetivo (satisfação) em relação ao currículo e ao professor, obteve-se a média total parcial de 4,65, para um desvio padrão de $\pm 0,39$. Tais resultados demonstram que os alunos demonstraram atitude majoritariamente positiva no que diz respeito ao componente afetivo do construto atitude quanto à Educação Física.

\subsection{Utilidade Percebida - componente cognitivo}

A avaliação das questões $6,7,10,13$ e 14 referentes ao componente cognitivo (utilidade percebida) em relação ao currículo, foi a que teve a menor média. Mesmo assim, os resultados obtidos também foram considerados positivos (média $=4,01$, desvio padrão $= \pm 0,84)$. $\mathrm{O}$ aspecto relacionado à utilidade do currículo mais presente nas entrevistas foi a saúde, como é possível verificar no relato do aluno P: "As atividades eram úteis na medida em que eu executava atividades físicas de um modo divertido, o que me tornava mais saudável de modo prazeroso, sem nenhum prejuízo". Também na percepção da aluna V: "Eu acho que eu emagrecia muito mais no laboratório do que na Educação Física e isso é importante pra mim". Seguindo esta lógica, o aluno G afirmou: "... não tenho vontade de fazer Educação Física, porque eu não gosto muito dos esportes tradicionais. Também porque é jogo e eu geralmente fico no gol, então [...] não é o que eu preciso da Educação Física. Tenho certeza que eu me movimentava muito mais jogando Xbox do que jogando na Educação Física real. Lá eu achava bem divertido e pra mim é importante, era uma maneira de eu praticar esportes fazendo uma coisa que eu goste”. Em sintonia com os relatos, Santos (2001) ao analisar a atitude de alunos em seu estudo, verificou que quase a totalidade destes considera o benefício à saúde como a finalidade principal da Educação Física.

A aluna A também faz um comentário a respeito: "Acho que a tecnologia é uma coisa que está influenciando tanto as nossas vidas hoje, tão dentro da gente. Todo 
mundo tem acesso e desde pequenas as crianças têm videogames e essas coisas, então eu acho que quanto mais a gente trouxer isso pra tornar o ambiente escolar um ambiente mais legal e mais prazeroso, melhor". Tal comentário vai diretamente ao encontro do que já afirmava Luepker (1999), que a tecnologia cada vez mais permeia o dia-a-dia das pessoas, especialmente dos jovens.

A análise das respostas para as questões 4, 8, 16, 17 e 18 referentes ao componente cognitivo (utilidade percebida) em relação ao professor, também demonstrou atitude positiva por parte dos alunos $(\mathrm{M}=4,33, \mathrm{DP}= \pm 0,79)$, ilustrada pela fala da aluna A: "Eu lembro que o professor inclusive nos falava curiosidades, algumas coisas que os colegas perguntavam". A aluna J também reforçou esta ideia: "O professor deu dicas não só do que fazer com o jogo, deu também dicas de musculação para os meninos, isso eu achei bem interessante".

Ao unificar os resultados das questões referentes ao componente cognitivo (utilidade percebida) em relação ao currículo e ao professor, obteve-se a média total parcial também apontando para uma atitude positiva dos alunos (média $=4,17$; desvio padrão $= \pm 0,81)$. Em se tratando deste aspecto, Zeng, Hipscher e Leung (2011) ao analisar a atitude de alunos do Ensino Médio constataram que estes em sua maioria acreditavam que a Educação Física beneficiaria a qualquer um que participasse dela regularmente. Dessa forma, tanto os resultados quantitativos, quanto as entrevistas do presente estudo, apontam para uma concordância com os achados destes autores.

Para obtenção dos resultados finais relativos ao construto atitude, computou-se a média de todas as respostas para as 20 questões. O resultado para a média foi de 4,41 , com $\mathrm{DP}= \pm 0,57$, apontando para uma atitude significativamente positiva em relação ao laboratório de exergames na Educação Física. A pontuação mais baixa dos estudantes que participaram da pesquisa foi de 3,6, pontuação que mesmo assim pode ser considerada elevada. Estes achados vão ao encontro dos resultados apresentados por Lwin e Malik (2012) que conduziram um estudo com o objetivo de avaliar a eficácia da incorporação de exergames nas aulas de Educação Física. Segundo os autores, incorporar exergames em aulas de Educação Física pode ser uma maneira efetiva de melhorar as percepções e comportamentos dos estudantes quanto às práticas de atividade física.

\section{Conclusão}

Este trabalho apresentou um estudo relativo à atitude de alunos em relação às aulas realizadas em um laboratório de exergames implementado em caráter experimental no ambiente escolar. A atitude dos alunos foi avaliada sob uma perspectiva duplo componente, considerando os aspectos cognitivo e afetivo. Os resultados alcançados demonstraram uma atitude positiva dos alunos tanto no que diz respeito ao componente cognitivo quanto afetivo.

A principal contribuição da pesquisa foi demonstrar que o laboratório experimental de exergames pode representar novo um espaço para desenvolvimento de práticas educacionais na Educação Física envolvendo tecnologia, em virtude da atitude positiva dos estudantes perante o mesmo. Os exergames têm-se mostrado um componente pertinente ao currículo da Educação Física escolar, tendo-se em vista a crescente necessidade de incorporação das tecnologias no cotidiano dos estudantes, o reclame destes por uma maior variedade nas atividades do currículo da disciplina, bem como a configuração deste tipo de jogo como novo elemento da cultura corporal de movimento que emerge da realidade concreta dos alunos.

$\mathrm{Na}$ literatura são poucos os relatos relacionados à utilização das percepções dos alunos na construção do currículo de Educação Física. Ao invés disso, o delineamento 
do currículo, incluindo a seleção e duração de implementação de cada conteúdo são comumente orientados pela teoria, e não por dados empíricos. Contudo, os resultados do estudo aqui apresentado demonstram a importância de considerar-se as vozes dos alunos para elaboração de propostas educacionalmente apropriadas e que possam aumentar a participação e motivação dos estudantes. Este estudo sugere a necessidade de atividades que tenham significado pessoal para os alunos, que estes se sintam participantes ativos no processo de aprendizagem. Sabe-se ainda que os estudantes apresentam diferentes atitudes em relação à Educação Física em decorrência de fatores como experiências anteriores na disciplina, nível de habilidade, gênero, idade, série escolar, classe social, etnia, entre outros (Subramanian e Silverman, 2007). Os resultados do presente estudo apresentam certas limitações na medida em que tais diferenças não foram consideradas na coleta e análise de dados. Novos estudos com maior número de participantes e estratificação de amostras podem possibilitar a realização destas avaliações.

\section{Referências}

Ajzen I. Attitudes, Personality, and Behavior. New York: Open University Press; 2005.

Baracho A.F.O., Gripp F.J., Lima M.R. Os Exergames e a Educação Física Escolar na Cultura Digital. Revista Brasileira de Ciências do Esporte. 2012;34(1):111-126.

Barg-Walkow et al. Understanding older adults' perceptions of and attitudes towards exergames. Gerontechnology. 2017, 16. p. 81-90.

Bryan C.L., Solmon M.A. Student motivation in physical education and engagement in physical activity. Journal of Sport Behavior. 2012; 35(3):267-285.

Chartzisarantis N., Hagger M., Biddle S., Smith B. The stability of the attitude-intention relationship in the context of physical activity. Journal of Sports Sciences. 2005; 23(1):49-61.

Entertainment Software Rating Board (ESRB). 2018. Disponível em: http://www.esrb.org/ratings/

Figley G.E. Determinants of Attitudes Toward Physical Education. Journal of Teaching in Physical Education. 1985; 4(4): 229-240.

Finco, M.D., Fraga, A.B. Rompendo fronteiras na Educação Física através dos videogames com interação corporal. Motriz. 2012; 8(3):533-541.

Gomwe H. Children's Attitudes Towards Physical Education in Selected Urban Primary Schools in Mutare - Zimbabwe [dissertação]. Alice (Zimbabwe): Universidade de Fort Hare, Programa de Pós-Graduação em Ciências do Movimento Humano; 2012.

Graves, L. Stratton, G., Ridgers, N.D., Cable, N.T. Energy expenditure in adolescents playing new generation computer games. British Medical Journal. 2007; 335:12821284.

Ho, S. S., Lwin, L., Sng, J.R.H., Yee, A.Z.H. Yee. Escaping through exergames: Presence, enjoyment, and mood experience in predicting children's attitude toward exergames. Computers in Human Behavior. 2017, 72. p. 381-389.

Luepker R.V. How physically active are American children and what can we do about it? International Journal of Obesity. 1999; 23(2):12-17.

Luke M.D., Sinclair G.D. Gender Differences in Adolescents' Attitudes Toward School Physical Education. Journal of Teaching in Physical Education. 1991;11:31-46. 
Lwin M. O., Malik S. The efficacy of exergames-incorporated physical education lessons in influencing drivers of physical activity: a comparison of children and preadolescents. Psychology of Sport and Exercise. 2012; 13(6):756-760.

Maddison $\mathrm{R}$ et al. Effects of active video games on body composition : a randomized. American Journal of Clinic Nutrition. 2011; 94(1):156-163.

Manley A.J., Whitaker L. Wii-learning: Using Active Video Games to enhance the learning experience of undergraduate sport psychology students. Sport \& Exercise Psychology Review. 2011; 7(2):45-55.

Mhurchu C. et al. Couch potatoes to jumping beans: a pilot study of the effect of active video games on physical activity in children. The international journal of behavioral nutrition and physical activity. 2008; 5(8).

Mozelius, P., Elggren, O., Clysén, J., Wiklund, M. Exergames in Secondary School Physical Education: Attitudes Amongst Swedish Students. Proceedings of the European Conference on e-Learning, ECEL. 2014.

Papastergiou M. Exploring the potential of computer and video games for health and physical education: A literature review. Computers \& Education. 2009; 53(3): 603-622.

Rikard L.G., Banville D. High school student attitudes about physical education. Sport, Education and Society. 2006; 11(4): 385-400.

Ryan S., Fleming D., Maina M. Attitudes of Middle School Students toward their Physical Education Teachers and Classes. Physical Educator. 2003; 60(2):28.

Santos F.C.M. Da Importância dos Conteúdos na Atitude dos Alunos Face à Aula de Educação Física [dissertação]. Porto (Lisboa): Universidade do Porto, Programa de PósGraduação em Desporto para Crianças e Jovens; 2001.

Sheehan D.P., Katz L. The Impact of a Six Week Exergaming Curriculum on Balance with Grade Three School Children using the. International Journal of Computer Science in Sport. 2012; 11(3): 5-22.

Staiano A.E., Calvert S.L. Exergames for Physical Education Courses: Physical, Social, and Cognitive Benefits. Child Development Perspective. 2011;5(2): 93-98.

Subramaniam, P.R., Silverman, S. Validation of Scores From an Instrument Assessing Student Attitude Toward Physical Education. Measurement in Physical Education and Exercise Science. 2000; 4(1):29-43.

Subramaniam P.R., Silverman S. Middle school students' attitudes toward physical education. Teaching and Teacher Education. 2007;23(5): 602-611.

Trout J., Christie B. Interactive Video Games in Physical Education: Rather than Contribute to a Sedentary Lifestyle, These Games Demand Activity from the Players. The Journal of Physical Education, Recreation \& Dance. 2007; 78(5): 29-35.

Vaghetti C.A.O., Mustaro P.N., Botelho S.S.C. Exergames no ciberespaço: uma possibilidade para Educação Física; 2011, 7 a 9 de novembro; Salvador, Brasil. Porto Alegre: SBC; 2011. p.1-10.

Zeng H.Z., Hipscer M., Leung R.W. Attitudes of high School Students toward Physical Education and Their Sport Activity Preferences. Journal of Social Sciences. 2011; 7(4):529-537. 